\title{
E-Health Tools to Improve Antibiotic Use and Resistances: A Systematic Review
}

\author{
Érico Carvalho ${ }^{1}$, Marta Estrela ${ }^{1}(0)$, Maruxa Zapata-Cachafeiro ${ }^{2,3}$, Adolfo Figueiras ${ }^{2,3,4}$, \\ Fátima Roque ${ }^{5,6}$ (D) and Maria Teresa Herdeiro ${ }^{1, *(\mathbb{D}}$ \\ 1 iBiMED-Institute of Biomedicine, Department of Medical Sciences, University of Aveiro, \\ 3800 Aveiro, Portugal; ericocarvalho@ua.pt (É.C.); mestrela@ua.pt (M.E.) \\ 2 Department of Preventive Medicine and Public Health, University of Santiago de Compostela, \\ 15702 Santiago de Compostela, Spain; maruxa.zapata@usc.es (M.Z.-C.); adolfo.figueiras@usc.es (A.F.) \\ 3 Consortium for Biomedical Research in Epidemiology and Public Health (CIBER Epidemiology and Public \\ Health-CIBERESP), 28001 Madrid, Spain \\ 4 Health Research Institute of Santiago de Compostela (IDIS), 15706 Santiago de Compostela, Spain \\ 5 Research Unit for Inland Development-Polytechnic of Guarda (UDI-IPG), 6300 Guarda, Portugal; \\ froque@ipg.pt \\ 6 Health Sciences Research Centre, University of Beira Interior (CICS-UBI), 6200 Covilhã, Portugal \\ * Correspondence: teresaherdeiro@ua.pt
}

Received: 10 July 2020; Accepted: 11 August 2020; Published: 12 August 2020

\begin{abstract}
Background: e-Health tools, especially in the form of clinical decision support systems (CDSSs), have been emerging more quickly than ever before. The main objective of this systematic review is to assess the influence of these tools on antibiotic use for respiratory tract infections. (2) Methods: The scientific databases, MEDLINE-PubMed and EMBASE, were searched. The search was conducted by two independent researchers. The search strategy was mainly designed to identify relevant studies on the effectiveness of CDSSs in improving antibiotic use, as a primary outcome, and on the acceptability and usability of CDSSs, as a secondary outcome. (3) Results: After the selection, 22 articles were included. The outcomes were grouped either into antibiotics prescription practices or adherence to guidelines concerning antibiotics prescription. Overall, 15 out of the 22 studies had statistically significant outcomes related to the interventions. (4) Conclusions: Overall, the results show a positive impact on the prescription and conscientious use of antibiotics for respiratory tract infections, both with respect to patients and prescribing healthcare professionals. CDSSs have been shown to have great potential as powerful tools for improving both clinical care and patient outcomes.
\end{abstract}

Keywords: clinical decision support system; CDSS; antimicrobial management; e-health

\section{Introduction}

After the appearance of antibiotics, common infections that previously caused death or illness started to be effectively treated [1]. However, in recent years, medical science has been challenged with the emergence of highly resistant bacterial strains dispersed across the world. This emergence is the result of several factors, such as worldwide travel activity and, especially, antibiotic misuse and overuse [2,3]. In the context of primary care, both antibiotic overuse and a lack of adherence to guidelines are prevalent, albeit underestimated, issues [4]. It has also been revealed that $20-50 \%$ of the total amount of antibiotics prescribed in intensive care are unnecessary or inappropriate [5].

Antibiotic overuse in respiratory tract infections is very evident [4]. Respiratory diseases, namely infections of the respiratory tract, are one of the leading causes of death and disability in the world and 
have been shown to have a high incidence [6,7]. These infections include common cold, pharyngitis, epiglottitis, and pneumonia. While symptomatic treatment is used for most viral infections, bacterial pneumonias are usually treated with antibiotics. However, while antibiotics are ineffective against viral pathogens [6] and should thus only be prescribed when secondary bacterial infections develop [8,9], a 2018 [4] study revealed that almost three-quarters of prescribed antibiotics were not prescribed according to guidelines, and only $11 \%$ of them were optimally prescribed.

While modern medicine constantly needs new types of antibiotics and antivirals to treat drug-resistant infections, the pipeline of new drugs is declining [10]. In order to compensate for the lack of new antibiotics, novel/innovative health tools may help to improve antibiotic use, with the aim of ultimately helping to control antimicrobial resistance. In the case of acute respiratory tract infections, probably the most impactful interventions involve the avoidance of the prescription of antibiotics for the treatment of conditions for which antibiotics use is not indicated. This is related to improving guideline adherence, since poor guideline adherence is one of the problems related to the inappropriate prescription of antibiotics [4,11].

Guideline adherence and healthcare quality can be improved using clinical decision support systems (CDSSs), which ultimately help to close the gap between optimal practice and actual clinical care. This is reflected in the reduction in medication errors [12,13]. Consider the fact that rules that are implicit in guidelines can be encoded into CDSSs, and clinical care pathways that have been shown to be difficult to implement in practices with low clinician adherence are now easier to put into practice [14]. CDSSs are information systems that directly aid in clinical decision-making about an individual, and are designed to improve clinical decision-making using multiple direct features, including guideline dissemination, alerts, reminders, drug dose calculations, reduction in antibiotics prescriptions, distribution, adequate consumption, and reduction in practice variation and error $[13,15,16]$. It also consists of software designed to be a direct aid in clinical decision-making. This software can allow information on a patient to be matched with a computerized database, and patient-specific recommendations can be presented to the clinician [17]. In more technically advanced systems, the characteristics of each patient are computerized, and the software algorithms generate specific recommendations.

Electronic health records (EHRs), computerized provider order entry systems (CPOEs), and CDSSs are powerful tools for providing a safer, more effective, and more efficient healthcare delivery [18]. That said, the use of information and communication technologies (ICTs) in health is transforming the provision and management of health care. ICT use can provide benefits not only in terms of obtaining health gains, but also in terms of monitoring, research, and demonstration, thus significantly contributing to the development of the knowledge and transparency of a system [19]. Health information technology (HIT) aims to improve the quality of care by optimizing the exchange and coordination of health care information, implementing state-of-the-art clinical practices and reducing medication errors and adverse events [12].

CDSSs can be seen as very useful tools for improving guideline adherence, particularly regarding antibiotic use for respiratory infections, especially when considering both the significant room for improvement and the high heterogeneity of clinical practices. With the increase in newly available information on antibiotic use, a multiplicity of modalities for educating and informing both patients and health professionals can be employed to supplement traditional educational methods [20]. The focus of this systematic review is to assess the influence of e-health tools, namely, CDSSs, on antibiotic use. Moreover, it will also evaluate the acceptability of e-health tools in relation to the prescription, dispensation and use of antibiotics by health professionals specifically for respiratory infections. 


\section{Materials and Methods}

\subsection{Protocol and Registration}

We followed the guidelines in the PRISMA Statement [21] in conducting this systematic review and recorded this study in the international database of prospectively registered systematic reviews (PROSPERO) (reg. no. CRD42020167316) [22].

\subsection{Search Strategy and Inclusion Criteria}

For this systematic review, searches in the scientific databases, MEDLINE-PubMed and EMBASE, on 4 February and 21 February 2020, respectively, were conducted.

The search was conducted by two independent researchers, and the search strategy was primarily designed to identify relevant studies on the effectiveness of CDSSs in improving antibiotic use, as a primary outcome, and on the acceptability and usability of CDSSs in the daily routine of health professionals, patients, and all users of these systems, as a secondary outcome. The following keywords and their equivalents were used in PubMed and EMBASE:

(clinical-decision-support-system OR decision-support-system OR computer-assisted decision-making OR expert-system OR decision-support) AND (antimicrobial resistance OR antimicrobial OR antibiotic $^{*}$ OR antimicrobial management) AND (electronic health OR e-health) NOT Tele-health.

The selection criteria applied in this review were as follows: (1) Language: the papers had to be published in English, Spanish, or Portuguese; (2) Condition or domain being studied: respiratory infections; (3) Type of outcome: studies had to describe the impact of e-health tools on antibiotic use; (4) Participants/population: health professionals and patients; (5) Types of study to be included: randomized and non-randomized (including cluster) trials and observational (including case-control, cross-sectional, cohort, before and after, and interrupted time series) studies. Study protocols, reviews, systematic reviews, and meta-analyses were excluded.

All titles resulting from the database searches were independently reviewed. The inclusion and exclusion criteria were applied by two independent researchers (E.C., M.E.), and subsequently validated by a third researcher (T.H.) in cases where there was no agreement.

\subsection{Quality Assessment of the Included Studies}

The quality of the included studies was assessed using a scale based on Garg's study, which comprises judgement and support for the judgement of each included study [23,24]. For each study, risk of bias and quality assessments were conducted separately by two researchers (EC, ME). In cases of disagreement, a third reviewer acted as a referee in order to reach a consensus (TH). The quality assessment was conducted based on five main characteristics, scored from 0-2:

(1) Allocation of study groups (random: 2, quasi-random: 1, selected controls: 0);

(2) Unit of allocation (cluster (such as a practice): 2, physician: 1, patient: 0);

(3) Baseline differences (presence of baseline differences with statistical adjustments: 2, baseline with no adjustments: 1 , no baseline differences: 0$)$;

(4) Objectivity of the outcome (blinded assessment: 2, no blinding but defined assessment criteria: 1 , no blinding and poorly defined: 0$)$;

(5) Completeness of follow-up (>90\%: 2, 80-90\%: 1, <80\% or not described: 0 ).

Each study was scored from 0 to 10 , based on the sum of the scores for each characteristic. Higher scores represent higher quality studies $[23,24]$.

\subsection{Data Extraction and Analysis}

The analyzed articles were summarized in two tables containing the author's information, date of publication, country, study design, population, source data, and outcomes, namely, whether the 
interventions had an impact on antibiotic use or not. The data were extracted independently by two researchers (EC, ME) and their assessments were compared. In cases of disagreement, a third and fourth reviewer $(\mathrm{TH}, \mathrm{FR})$ acted as referees in order to reach a consensus.

\section{Results}

\subsection{Study Selection}

After extracting all 498 articles from the databases, the eligible articles were selected based on the title and abstract. The inclusion criterion of the studies was the impact of the e-health tools on antibiotics use for respiratory tract infections, namely, antibiotics prescription, antibiotics consumption, and adherence to guidelines on antibiotics prescription. All studies that mentioned this impact were considered. After the selection, based on the title, abstract, and review of duplicates, 79 full-text articles were assessed, of which 22 were considered eligible to be included in the present review [25-47] (Figure 1).
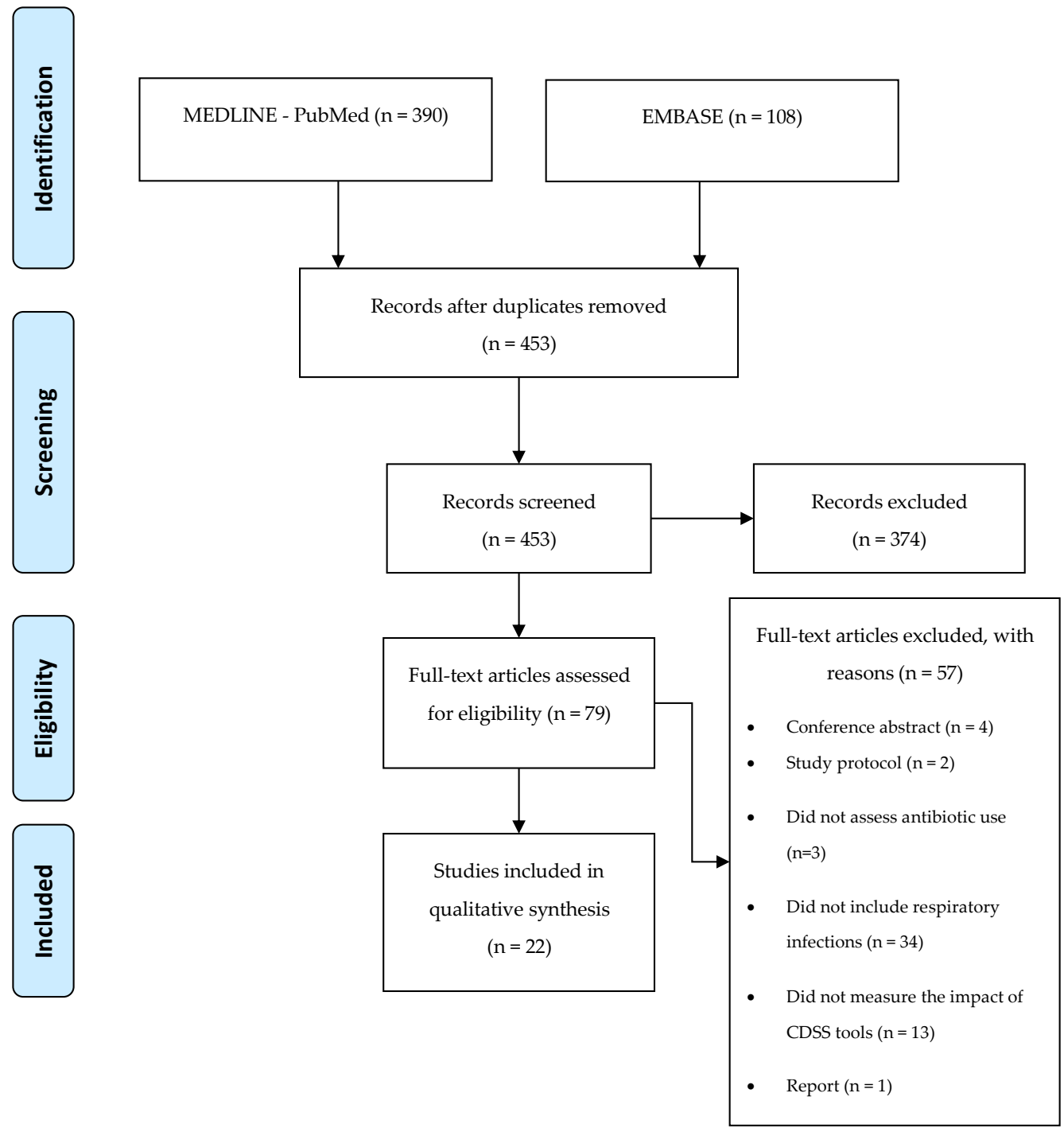

Figure 1. Application of search strategies to retrieve the total number of studies for analysis. 


\subsection{Quality Assessment}

All of the studies were evaluated regarding their quality. The average score for all the studies was 5.57. Seven studies had a score of $4 / 10$ or below $[28,32,35,38,40,43,46]$. More than half of the studies $(54.5 \%)$ had a total score between five and seven [25-27,29-31,33,34,36,37,39,47]. Three other studies had a score above seven $[41,44,45]$. The results are presented in Table S1.

\subsection{Study Characteristics}

Information on the study design, location, setting, study population, diseases, and outcomes assessed were retrieved from the included articles. This section sums up the study characteristics of the included papers (Table 1).

\subsubsection{Study Design}

Of the 22 included studies, $8(36.3 \%)$ were randomized controlled trials, which is the gold standard for intervention effect assessment $[25,27,32,33,36,41,44,45]$. Six other studies were pre-post studies $(27.2 \%)$ [29-31,40,43,46]. Five studies $(22.7 \%)$ were retrospective studies [28,34,38,47]. Two observational studies that did not specify the type of study design were also considered $[37,39]$, as well as one study with a mixed-methods design [35].

\subsubsection{Location}

Most studies (81.8\%) took place in the USA [25-34,36-41,46,47]. Three studies (13.6\%) were undertaken in the UK [35,44,45], and one was conducted in Australia (4.5\%) [43].

\subsubsection{Setting}

Most of the studies (68.1\%) took place in primary care/ambulatory practices [27-31,34-37,39,41,44-46]. Five of the interventions (22.7\%) occurred in the context of hospital care [26,38,40,43,47], and 9.0\% occurred in academic medical centers $[32,33]$. The remaining interventions occurred in a pediatric practice [25].

\subsubsection{Study Population}

Sixteen out of the 22 included studies considered the results obtained based on the entire population [26,28-30,32-39,41,43,46,47]. Four studies only measured the adult population [31,40,44,45]. One study only considered children and adolescents [25], and one other study did not assess the impact on the population at all [27].

\subsubsection{Diseases}

A total of 13 out of the 22 articles assessed antibiotic use related to respiratory tract infections/acute respiratory diseases $[25-31,34,35,38,39,44,45]$. Three studies measured the impact of CDSS tools associated with pneumonia $[40,43,47]$. Three studies considered both streptococcal pharyngitis and pneumonia [32,33], and one article evaluated clinical cases of sinusitis and pharyngitis [46]. Two other studies took into account either sinusitis [37] or uncomplicated acute bronchitis [41].

\subsubsection{Intervention}

Eight interventions studied CDSSs that were used to aid in the diagnosis of respiratory diseases $[25,30,32,33,36,37,41,46]$, while ten studies focused on the treatment of these diseases [26,28,34,35,38,40,43-45,47]. Four other studies covered both diagnosis and treatment $[27,29,31,39]$. Most of the studies consisted of forms/templates/algorithms that provided a final recommendation based on the information that the health professionals provided to the system $[25-33,36,39,40,43,46,47]$. However, seven studies operated by providing educational material or alerts on clinical practice $[34,35,37,38,41,44,45]$. 
Table 1. Synthesis of the studies' characteristics and respective outcomes.

\begin{tabular}{|c|c|c|c|c|c|c|c|}
\hline Author (Year) & Title & Study Design & Location & Setting & Disease & Study Population & Intervention \\
\hline Bourgeois FC (2010) [25] & $\begin{array}{l}\text { Impact of a computerized template on } \\
\text { antibiotic prescribing for acute respiratory } \\
\text { infections in children and adolescents. }\end{array}$ & RCT & USA & Pediatric practice & RTI & Children and Adolescents & $\begin{array}{l}\text { Template for diagnosis with } \\
\text { clinical support }\end{array}$ \\
\hline Gifford J (2017) [26] & $\begin{array}{c}\text { Decision support during electronic } \\
\text { prescription to stem antibiotic overuse for } \\
\text { acute respiratory infections: a long-term, } \\
\text { quasi-experimental study. }\end{array}$ & Retrospective study & USA & Hospital care & RTI & ALL & $\begin{array}{l}\text { CDSS deployed at the } \\
\text { moment of AB prescription }\end{array}$ \\
\hline Ginzburg R (2018) [37] & $\begin{array}{l}\text { Using Clinical Decision Support Within the } \\
\text { Electronic Health Record to Reduce } \\
\text { Incorrect Prescribing for Acute Sinusitis. }\end{array}$ & Observational cohort & USA & Primary care clinics & Sinusitis & ALL & Best practice alert \\
\hline Gonzales R (2013) [41] & $\begin{array}{l}\text { A cluster randomized trial of decision } \\
\text { support strategies for reducing antibiotic } \\
\text { use in acute bronchitis. }\end{array}$ & CRCT & USA & Primary care clinics & $\begin{array}{l}\text { Uncomplicated } \\
\text { acute bronchitis }\end{array}$ & ALL & Best practice alert \\
\hline Grayson ML (2004) [43] & $\begin{array}{l}\text { Impact of an electronic antibiotic advice } \\
\text { and approval system on antibiotic } \\
\text { prescribing in an Australian } \\
\text { teaching hospital. }\end{array}$ & $\begin{array}{l}\text { Prospective, } \\
\text { Non-randomized: } \\
\text { Pre/post-study }\end{array}$ & Australia & Hospital care & CAP & ALL & $\begin{array}{c}\text { Computer-generated } \\
\text { AB approval }\end{array}$ \\
\hline Gulliford MC (2014) [44] & $\begin{array}{l}\text { Electronic health records for intervention } \\
\text { research: a cluster randomized trial to } \\
\text { reduce antibiotic prescribing in primary } \\
\text { care (eCRT study). }\end{array}$ & RCT & UK & Primary care clinics & RTI & Adult & $\begin{array}{l}\text { CDSS with education and } \\
\text { decision support }\end{array}$ \\
\hline Gulliford MC (2019) [45] & $\begin{array}{l}\text { Effectiveness and safety of electronically } \\
\text { delivered prescribing feedback and } \\
\text { decision support on antibiotic use for } \\
\text { respiratory illness in primary care: } \\
\text { REDUCE cluster randomized trial. }\end{array}$ & CRCT & UK & Primary care clinics & RTI & Adult & $\begin{array}{l}\text { Webinar }+\mathrm{AB} \text { reports }+ \\
\text { Decision support tools }\end{array}$ \\
\hline Hingorani R (2015) [46] & $\begin{array}{l}\text { Improving antibiotic adherence in } \\
\text { treatment of acute upper respiratory } \\
\text { infections: a quality improvement process. }\end{array}$ & $\begin{array}{l}\text { Prospective, } \\
\text { Non-randomized: } \\
\text { Pre/post-study }\end{array}$ & USA & Primary care clinics & Sinusitis, pharyngitis & ALL & $\begin{array}{l}\text { Didactic teaching, AB } \\
\text { guidelines, CDSS integrated } \\
\text { on EHR }\end{array}$ \\
\hline Jones BE (2018) [47] & $\begin{array}{c}\text { In Data We Trust? Comparison of } \\
\text { Electronic Versus Manual Abstraction of } \\
\text { Antimicrobial Prescribing Quality Metrics } \\
\text { for Hospitalized Veterans } \\
\text { with Pneumonia. }\end{array}$ & Retrospective study & USA & Hospital care & Uncomplicated pneumonia & ALL & $\begin{array}{l}\text { Electronic vs. manual } \\
\text { Medication Use } \\
\text { Evaluation (MUE) }\end{array}$ \\
\hline Linder J (2007) [29] & $\begin{array}{l}\text { Clinical decision support to improve } \\
\text { antibiotic prescribing for acute respiratory } \\
\text { infections: results of a pilot study. }\end{array}$ & $\begin{array}{l}\text { Prospective, } \\
\text { Non-randomized: } \\
\text { Pre/post-study }\end{array}$ & USA & Primary care clinics & RTI & ALL & $\begin{array}{l}\text { ARI Smart Form: assistance } \\
\text { in AB prescription for } \\
\text { RTI visits }\end{array}$ \\
\hline Linder JA (2006) [28] & $\begin{array}{l}\text { Acute infections in primary care: accuracy } \\
\text { of electronic diagnoses and electronic } \\
\text { antibiotic prescribing. }\end{array}$ & $\begin{array}{l}\text { Retrospective study, } \\
\text { (double) cross-sectional }\end{array}$ & USA & Primary care clinics & RTI & ALL & Use of electronic prescribing \\
\hline
\end{tabular}


Table 1. Cont.

\begin{tabular}{|c|c|c|c|c|c|c|c|}
\hline Author (Year) & Title & Study Design & Location & Setting & Disease & Study Population & Intervention \\
\hline Linder JA (2009) [27] & $\begin{array}{l}\text { Documentation-based clinical decision } \\
\text { support to improve antibiotic prescribing } \\
\text { for acute respiratory infections in primary } \\
\text { care: a cluster randomized controlled trial. }\end{array}$ & CRCT & USA & Primary care clinics & RTI & n.m. & $\begin{array}{l}\text { ARI Smart Form: assistance in } \\
\text { AB prescription for RTI visits }\end{array}$ \\
\hline Litvin CB (2013) [30] & $\begin{array}{l}\text { Use of an electronic health record clinical } \\
\text { decision support tool to improve antibiotic } \\
\text { prescribing for acute respiratory infections: } \\
\text { the ABX-TRIP study. }\end{array}$ & $\begin{array}{l}\text { Prospective, } \\
\text { Non-randomized: } \\
\text { Pre/post study }\end{array}$ & USA & Primary care clinics & RTI & ALL & $\begin{array}{l}\text { ABX-TRIP: guidelines, } \\
\text { diagnostic criteria, AB } \\
\text { use recommendation }\end{array}$ \\
\hline Mainous AG (2013) [31] & $\begin{array}{l}\text { Impact of a clinical decision support } \\
\text { system on antibiotic prescribing for acute } \\
\text { respiratory infections in primary care: } \\
\text { quasi-experimental trial. }\end{array}$ & $\begin{array}{l}\text { Prospective, } \\
\text { Non-randomized: } \\
\text { Pre/post-study }\end{array}$ & USA & Primary care clinics & RTI & Adult & $\begin{array}{l}\text { CDSS on EHR, helps with } \\
\text { appropriate diagnosis and } \\
\text { AB suggestions }\end{array}$ \\
\hline Mann D (2014) [32] & $\begin{array}{l}\text { Measures of user experience in a } \\
\text { streptococcal pharyngitis and pneumonia } \\
\text { clinical decision support tools. }\end{array}$ & RCT & USA & Academic center & $\begin{array}{l}\text { streptococcal pharyngitis } \\
\text { and pneumonia }\end{array}$ & ALL & $\begin{array}{l}\text { CDSS tool (iCPR) with } \\
\text { Smartset (medication } \\
\text { bundled-order set) }\end{array}$ \\
\hline McCullagh LJ (2014) [33] & $\begin{array}{c}\text { User centered clinical decision support } \\
\text { tools: adoption across } \\
\text { clinician training level. } \\
\end{array}$ & RCT & USA & $\begin{array}{l}\text { Academic medical } \\
\text { institution }\end{array}$ & $\begin{array}{l}\text { streptococcal pharyngitis } \\
\text { and pneumonia }\end{array}$ & ALL & $\begin{array}{l}\text { CDSS tool (iCPR) with } \\
\text { Smartset (medication } \\
\text { bundled-order set) }\end{array}$ \\
\hline McCullough JM (2014) [34] & $\begin{array}{l}\text { Impact of clinical decision support on } \\
\text { receipt of antibiotic prescriptions for acute } \\
\text { bronchitis and upper respiratory } \\
\text { tract infection. }\end{array}$ & Retrospective study & USA & Primary care clinics & RTI & ALL & CDSS use assessment \\
\hline McDermott L (2014) [35] & $\begin{array}{l}\text { Process evaluation of a point-of-care } \\
\text { cluster randomised trial using a } \\
\text { computer-delivered intervention to reduce } \\
\text { antibiotic prescribing in primary care. }\end{array}$ & Mixed methods & UK & Primary care clinics & RTI & ALL & Computer point-of-care \\
\hline McGinn TG (2013) [36] & $\begin{array}{l}\text { Efficacy of an evidence-based clinical } \\
\text { decision support in primary care practices: } \\
\text { a randomized clinical trial. }\end{array}$ & RCT & USA & Primary care clinics & $\begin{array}{l}\text { Streptococcal pharyngitis and } \\
\text { pneumonia. }\end{array}$ & ALL & Clinical prediction tool \\
\hline Rattinger GB (2012) [38] & $\begin{array}{l}\text { A sustainable strategy to prevent misuse of } \\
\text { antibiotics for acute respiratory infections. }\end{array}$ & Retrospective study & USA & Hospital care & RTI & ALL & $\begin{array}{l}\text { CDSS with treatment paths } \\
\text { for fluoroquinolones } \\
\text { and azithromycin }\end{array}$ \\
\hline Rubin MA (2006) [39] & $\begin{array}{l}\text { Use of a personal digital assistant for } \\
\text { managing antibiotic prescribing for } \\
\text { outpatient respiratory tract infections in } \\
\text { rural communities. }\end{array}$ & $\begin{array}{l}\text { Observational } \\
\text { randomized study }\end{array}$ & USA & Primary care clinics & RTI & ALL & $\begin{array}{l}\text { CDSS with diagnostic and } \\
\text { therapeutic recommendation }\end{array}$ \\
\hline Webb BJ (2019) [40] & $\begin{array}{l}\text { Antibiotic Use and Outcomes After } \\
\text { Implementation of the Drug Resistance in } \\
\text { Pneumonia Score in ED Patients With } \\
\text { Community-Onset Pneumonia. }\end{array}$ & $\begin{array}{l}\text { Prospective, } \\
\text { Non-randomized: } \\
\text { Pre/post-study }\end{array}$ & USA & Hospital care & Pneumonia & Adult & DRIP score calculator \\
\hline
\end{tabular}

AB-Antibiotic; ARI-Acute Respiratory Infection; CAP—Community-acquired pneumonia; CDSS—Clinical decision support system; DRIP—Drug-Resistance in Pneumonia;

EHR—Electronic health records; RTI—Respiratory tract infections; (C)RCT—(Cluster) Randomized controlled trial; n.m. - not mentioned. 


\subsubsection{Outcomes}

The outcomes were classified into two main groups: (1) antibiotics prescription practices, and (2) adherence to guidelines concerning antibiotics prescription. Overall, 14 out of the 22 studies $(63.6 \%)$ had statistically significant outcomes related to the interventions [26,30-32,36-41,44-47]. Five studies did not assess statistical significance [28,29,34,35,43]. Two studies were not statistically significant $[27,33]$. One study did not obtain statistically significant outcomes from comparing the control group with the intervention group. However, as the e-health tool was not used on all eligible visits to the intervention group, the study showed statistically significant differences in terms of prescription when comparing the visits in which the CDSS was used with the ones in which it was not used [25].

Seventeen studies (77.2\%) assessed the impact of e-health tools on antibiotic prescription [25,27-37,40,41,44,45,47], two of which did not have statistically significant results [27,33]. Fifteen studies showed an impact of e-health tools on antibiotic prescription [25,28-32,34-37,40,41,44,45,47]. Overall, the studies showed positive results on antibiotics prescription of improving the quality or reducing the number of antibiotics prescriptions. However, some heterogeneity in the strength of the effectiveness of CDSSs can also be noted, as some studies show modest, albeit positive and significant, results. One article simultaneously evaluated both the acceptance of the tool used in the intervention and its impact on antibiotic prescription [33]. However, while the tool acceptance was statistically significant, the impact on prescriptions was not [33]. The results are summarized in Table 2.

Table 2. Summary of the included studies' results on antibiotic prescription.

\begin{tabular}{|c|c|c|c|c|}
\hline Author (Year) & Population (n) & Results & $p$-Value/CI & Observations \\
\hline Bourgeois FC (2010) [25] & $\begin{array}{l}C=12 \\
P=146 \\
V=419\end{array}$ & $\begin{array}{l}\text { (1) Intervention group vs. control group: } 39.7 \% \text { vs. } \\
46 \% \text { prescription rate; * } \\
\text { (2) Intervention group: with ARI-IT users vs. } \\
\text { non-ARI-IT users: } 31.7 \% \text { vs. } 39.9 \% \text { prescription rate. }\end{array}$ & $\begin{array}{l}\text { (1) } p=0.844 ; * \\
\text { (2) } p=0.02\end{array}$ & $\begin{array}{l}\text { Usability: ARI-IT likely to } \\
\text { improve efficiency }\end{array}$ \\
\hline Ginzburg R (2018) [37] & $\begin{aligned} \mathrm{P} & =54 \\
\mathrm{~V} & =438\end{aligned}$ & $\begin{array}{l}\text { (1) Prescription reduction: } 86.3 \% \text { to } 61.7 \% \text {; } \\
\text { (2) Incorrect prescription: } 88.5 \% \text { to } 78.7 \% \text {. }\end{array}$ & $\begin{array}{l}\text { (1) } p<0.01 \\
\text { (2) } p=0.02\end{array}$ & \\
\hline Gonzales R (2013) [41] & $\begin{array}{c}C=12 \\
P=155 \\
V=12826\end{array}$ & Prescription reduction: $74.3 \%$ to $60.7 \%$ & $p=0.014$ & \\
\hline Gulliford MC (2014) [44] & $\begin{array}{c}\mathrm{C}=100 \\
\mathrm{~V}=603409\end{array}$ & Prescription reduction by $9.69 \%$. & $p=0.034$ & \\
\hline Gulliford MC (2019) [45] & $C=79$ & $\begin{array}{l}\text { Prescription intervention group } v s \text {. control group } \\
\qquad(\mathrm{RR}=0.88) .\end{array}$ & CI $(0.78-0.99) ; p=0.040$ & $\begin{array}{l}\text { No effect in children }<15 \\
\text { years and adults }>84 \text { years }\end{array}$ \\
\hline Jones BE (2018) [47] & $\begin{array}{c}C=30 \\
P=111 \\
V=2004\end{array}$ & $\begin{array}{l}\text { Evaluations as excessive } \mathrm{AB} \text { duration: } \\
\quad \mathrm{mMUE}=82.3 \%, \mathrm{eMUE}=84.0 \%\end{array}$ & $p<0.001$ & \\
\hline Linder JA (2009) [27] & $\begin{array}{c}C=27 \\
P=443 \\
V=21961\end{array}$ & $\begin{array}{l}\text { Prescription rate: Intervention group }=39 \% \text { vs. } \\
\text { control group }=43 \%(\mathrm{OR}=0.8)^{*}\end{array}$ & $\mathrm{CI}(0.5-1.3)^{*}$ & \\
\hline Litvin CB (2013) [30] & $\begin{array}{l}\mathrm{C}=9 \\
\mathrm{Ph}=27 \\
\mathrm{~N}=6 \\
\mathrm{~A}=6\end{array}$ & $\begin{array}{l}\text { (1) Inappropriate AB use: }+1.57 \% \text { *, } \\
\text { (2) Broad spectrum AB use: } \\
-16.30 \%\end{array}$ & $\begin{array}{l}\text { (1) CI }(-5.35 \%, 8.49 \%)^{*} \\
\text { (2) CI }(-24.81 \%,-7.79 \%)\end{array}$ & \\
\hline Mainous AG (2013) [31] & $C=70$ & $\begin{array}{l}\text { (1) Inappropriate } \mathrm{AB} \text { use: Intervention group } v s \text {. } \\
\text { control group: }-0.6 \% /+4.2 \% / \text {; } \\
\text { (2) Broad-spectrum AB use: Intervention group vs. } \\
\text { control group: } \\
-16.6 \% /+1.2 \%\end{array}$ & $\begin{array}{l}\text { (1) } p=0.03 \\
\text { (2) } p<0.0001\end{array}$ & \\
\hline
\end{tabular}


Table 2. Cont.

\begin{tabular}{|c|c|c|c|c|}
\hline Author (Year) & Population (n) & Results & $p$-Value/CI & Observations \\
\hline McCullough JM (2014) [34] & $\mathrm{V}=3317$ & $\begin{array}{l}\text { Use of CDSS associated with a 19\% lower } \\
\text { likelihood of prescription }\end{array}$ & - & \\
\hline McDermott L (2014) [35] & $\begin{array}{l}\mathrm{C}=100 \\
\mathrm{P}=103\end{array}$ & System could decrease $\mathrm{AB}$ prescription rates & - & Useful features of CDSS \\
\hline McGinn TG (2013) [36] & $\mathrm{V}=984$ & $\begin{array}{l}\mathrm{AB} \text { prescription: intervention group vs. control } \\
\text { group }(\mathrm{RR}=0.74)\end{array}$ & CI $(0.60-0.92)$ & \\
\hline Webb BJ (2019) [40] & $V=2169$ & Broad-spectrum antibiotic use $(\mathrm{OR}=0.62)$ & $\mathrm{CI}(0.39-0.98), p=0.039$ & \\
\hline $\begin{array}{l}\text { C-Clinics/Practic } \\
\text { EHR-Electronic } \\
\text { PGY-Post-graduc } \\
\text { OR-Odds ratio; I }\end{array}$ & $\begin{array}{l}\text { P-Provic } \\
\text { health recor } \\
\text { e year; MUE- } \\
\text { R-Risk ratio }\end{array}$ & $\begin{array}{l}\mathrm{V} \text {-Visits/Cases/Patients; Ph-Pl } \\
\text { CDSS-Clinical decision suppo } \\
\text { edical use evaluation; ARI-IT-Acut } \\
\text {-Confidence interval; }{ }^{*} \text { - not statisti }\end{array}$ & $\begin{array}{l}\text { sicians; } \mathrm{N}-\mathrm{Nurse} \\
\text { system; A-Phy } \\
\text { Respiratory Illness It } \\
\text { lly significant. }\end{array}$ & $\begin{array}{l}\text { AB-Antibiotic; } \\
\text { rian Assistants; } \\
\text { ractive Template; }\end{array}$ \\
\hline
\end{tabular}

Five studies $(21.7 \%)$ evaluated the impact of e-health tools on the adherence to guidelines/prescription congruence and adequacy, out of which four had statistically significant improvements on this outcome $[26,38,39,46]$. One study did not assess statistical significance, although it had positive results [43]. The results reflect an overall improvement in guideline concordance. Guideline adherence also improved when CDSS tools were used more than once [39]. One study also assessed the impact of CDSS withdrawal, observing an improvement in guideline-discordant antibiotic use, which reinforced the positive impact of CDSSs on guideline adherence [26]. The results are summarized below in Table 3.

Table 3. Summary of the included studies' results on guideline concordance/adherence.

\begin{tabular}{|c|c|c|c|c|}
\hline Author (Year) & Population (n) & Results & $p$-Value/CI & Observations \\
\hline Gifford J (2017) [26] & $\mathrm{V}=1131$ & $\begin{array}{l}\text { Adjusted odds of guideline } \\
\text { concordance vs. "all other antibiotics": } \\
\text { - } \quad \text { Azithromycin }(\mathrm{OR}=8.8), \\
\text { - } \quad \text { Gatifloxacin }(\mathrm{OR}=24.4), \\
\text { - } \quad \text { Fluoroquinolone }(\mathrm{OR}=5.5)\end{array}$ & $\begin{array}{l}\text { CI Az (5.7-13.6); } \\
\text { CI GT (9.0-66.3); } \\
\text { CI Fl (CI 3.5-8.8) }\end{array}$ & \\
\hline Grayson ML (2004) [43] & $V=2000$ & $\begin{array}{l}\text { Exact concordance/concordance in } \\
76 \% \text { of the cases }\end{array}$ & - & \\
\hline Hingorani R (2015) [46] & $\begin{array}{c}\mathrm{Ph}=27 \\
\mathrm{~N}=1 \\
\mathrm{~V}=240\end{array}$ & $\begin{array}{l}\text { Intervention group }=91.25 \% \text { vs. } \\
\quad \text { control group }=78.6 \%\end{array}$ & $p<0.001$ & Usage rate: $40.5 \%$ \\
\hline Rattinger GB (2012) [38] & $\mathrm{V}=3831$ & Congruent prescription $(\mathrm{RR}=2.57)$ & CI (1.865-3.540) & \\
\hline Rubin MA (2006) [39] & $\mathrm{V}=14393$ & $82 \%$ adherence to CDSS, $2.7 \%$ change & $p=0.016$ & $\begin{array}{l}\text { Usability score of } \\
4.6 \text { (on a } 1-5 \text { scale) }\end{array}$ \\
\hline
\end{tabular}

Some studies also evaluated the acceptability and/or usability of the tools used in the interventions $[25,32,35,39,46]$. Overall, clinicians' perceptions emphasized the usefulness of the systems with positive opinions [25,35]. In Hingorani's study, the system was used in $40.5 \%$ of the visits [46]. In one study, the system scored highly in terms of usability, presenting very positive results [39]. Regarding Mann's intervention, a heterogeneity in the acceptance of the system components can be observed, with higher acceptance rates towards the lower risk of strep throat or pneumonia diagnoses and lower rates in higher risk diagnoses. When considering diagnoses and antibiotics in combination, only $14 \%$ completed the Smartset order [32].

\section{Discussion}

Overall, it appears that e-health tools have a positive impact both on the prescription and conscientious use of antibiotics in relation to respiratory tract infections for prescribing healthcare professionals. However, almost a third of the studies did not present statistically significant results [27,33] or did not assess statistical significance at all [28,29,34,35,43]. Considering all of the 22 included studies, it can be seen that the tools are mainly focused on antibiotics prescription 
practices, which generally resulted in positive outcomes, whether as a consequence of improving the quality of prescription or reducing the overall number of antibiotics prescriptions. This significantly positive impact on the quality of antibiotics prescription is in agreement with the literature, either in the context of primary [48] or hospital care [49]. CDSS tools have been shown to be effective in improving antibiotics prescription in primary care, and hospitals have been increasingly adopting electronic medical record systems, which have allowed for the emergence of new opportunities in integrating antimicrobial hospital policies, decision support, and antimicrobial usage and surveillance. However, further high-quality research in both contexts should be conducted in order to consistently assess the impact of these tools on clinical practices [48,49].

It is interesting to note that the literature emphasizes that different study designs answer different questions, and researchers should choose the most appropriate study design to evaluate CDSS tools according to their setting [50]. While randomized controlled trials, as well as other experimental designs, are adequate for studying specific changes in clinical practice behaviors, Kaplan et al. [50] argue that they might not suit investigations on other issues, such as the effects associated with whether or not systems are used [50]. Rawson et al. [51] argue that the study designs used to investigate these interventions usually require a standardized view of CDSSs, involving essentially the selection of heterogenous and non-standardized outcomes. These outcomes, namely, the total number of antimicrobial prescriptions, do not directly measure clinical outcomes, such as mortality, adverse events, and the development of antimicrobial resistance, which might constitute a problem in measuring the overall effectiveness of these tools [51].

The selected studies in which the intervention measured antibiotic prescription as the main outcome displayed some discrepancies associated with the significance of their results. In Bourgeois' study [25], no significant difference was detected in the total antibiotic prescriptions between clinicians in both the intervention and control groups. However, when the authors took into consideration the fact that most of the participants in the intervention group did not use the CDSS tool as expected, it became evident that the ones who used it had significantly reduced the total antibiotic prescriptions [25]. For this reason, user behavior appears to be an important outcome to assess in connection with this type of intervention, since it might have an important impact on the obtained results.

Regarding guideline adherence, e-health tools have been shown to have a positive effect in all considered studies, which is congruent with the literature $[17,23]$. However, despite these positive results, it is important to note that one of these studies did not assess the statistical significance of guideline concordance [43].

These alterations on clinical practices arising from the increased use of e-health tools, especially regarding antibiotic prescription quantity and quality, may ultimately reduce several problems associated with inadequate antibiotic use, namely, antibiotic resistances. In general, the quality of healthcare constitutes the major facilitator of e-health interventions, while costs are the major barrier [52]. For this reason, researching the implementation of these tools in a clinical and real context, thus allowing for a realistic assessment of their influence on clinical practice, is essential.

Studies highlighting the usability of these tools are emerging, and several scales of the usability of e-health tools have recently been published [53]. These types of studies, as well as acceptability studies, allow for a more profound analysis of the role of e-health tools in a clinical context. It is also very important that studies provide detailed information not only on the intervention's methodology per se, but also on e-health tools, in order to improve reproducibility and allow for similar research in other contexts. The challenge of designing information systems for a domain as complex as healthcare should be recognized. Few guidelines exist that aim to allow developers to follow common, effective, and safe practices, but significant advances can be achieved by focusing on human factors and a user-centered design, as the tools are built in consideration of the user, instead of forcing the adaptation to an idealized tool [32,54]. Despite the impact that user-centered designs have on the acceptance of e-health tools, only three studies $[32,33,36]$ considered a user-centered design for their e-health tools, which might constitute an obstacle to the optimization of the remaining systems. 
Some studies also assessed the usability and acceptance of the e-health tools used in the interventions $[25,32]$. The user reports given by clinicians were positive, and it is widely believed that these tools can strongly improve clinical practice and aid in the improvement of the quality of antibiotics prescription $[25,32,33,35,39]$. Health professionals also emphasized the ease of use of these tools. However, it is important to note that each of these e-health tools may have a different learning curve, based on their intrinsic complexity and the overall familiarity that health professionals have with these types of software. Younger physicians also appeared to accept e-health tools more easily than older practitioners. Health professionals with higher levels of training appeared to be less accepting of CDSSs. For this reason, CDSS engagement should be tailored based on age and training level to improve usability and acceptance [33].

Based on Sirajuddin's study [55], modern CDSSs should adhere to key principles, like the CDS Five Rights model. This model suggests that sustainable improvements are more likely if they communicate "the right information to the right person, in the right format, through the right channel, and at the right time" [55]. Conducting implementation research on this topic, focusing mainly on implementation issues associated with CDSS tools, with the main objective of supporting and promoting successful interventions that have been demonstrated to be effective, is highly important [56]. Despite its relevance, it is a somewhat neglected field of study, either due to a lack of investment in implementation research activities or a lack of overall understanding on what implementation research has to offer. Despite the high investment in health innovation, research that considers how innovative tools can be better used and implemented has not attracted significant funding [56]. This might be the reason why a vast majority of e-health interventions tend to fail clinical implementation, despite displaying promising research results [52]. In order to attain a successful outcome, the assessment of e-health interventions should be based essentially on three pillars of care: access, quality, and cost containment [52].

This study has various strengths, namely, the extensive and systematic research of articles on the stated topic. However, one of the limitations of this paper is its use of only two databases (PubMed and EMBASE), which may have led to a lack of consideration of other potentially relevant articles on other databases. Given the heterogeneity of methods, interventions, and outcomes, a meta-analysis of the effectiveness of the interventions could not be performed. This heterogeneity also brings some barriers to the drawing of some conclusions based solely on the outcomes, considering the differences between the results obtained, for instance, regarding guideline adherence. Another limitation is associated with the timeline of some of these studies, since they are more than ten years old, and at that time, CDSS tools were not as prevalent as they are nowadays.

After the quality assessment, it can be noted that some studies might present an overall higher bias risk. The acquisition of a low average score (5.57) may indicate that studies on the effect of CDSSs on antibiotics have a poorer methodological quality when compared to studies on other drug groups or other types of therapeutic interventions $[23,24]$.

While CDSS tools have an overall potential to be powerful in enhancing clinical care while, at the same time, offering a promising future for optimizing antibiotic prescription, it may be difficult to generalize, as the vast majority of the studies were conducted in the United States, and they may therefore not reflect the diversity of healthcare worldwide regarding clinical practice, prescription behaviors, and even policies on antibiotics use [57].

\section{Conclusions}

This review indicates that interventions using e-health tools, especially CDSSs, can be effective in optimizing and reducing antibiotics prescription. However, it is pertinent to emphasize that the outcomes measured were highly heterogenous and expressed different levels of effectiveness. For this reason, this review only allows for an overall picture of the potential that CDS tools have in relation to antibiotics use. The CDSS interventions themselves were also highly heterogenous, having different approaches concerning antibiotics use, e.g., some tools focused on reducing antibiotic prescriptions, while others turned to guideline adherence or improving the quality of antibiotics 
prescriptions. Despite these limitations, the included studies revealed that health professionals are very receptive to the use of e-health tools. Antibiotic prescription is a particularly complex area in medical decision-making, so further research is required to determine the characteristics of CDSSs, which are crucial for obtaining a high guideline concordance. The conclusions of this review can be used to enrich the debate on the impact of CDSSs on antibiotic optimization.

Supplementary Materials: The following are available online at http://www.mdpi.com/2079-6382/9/8/505/s1, Table S1: Quality assessment results.

Author Contributions: Conceptualization: M.T.H., F.R., A.F.; methodology: M.T.H., F.R., A.F.; validation: M.T.H., F.R., A.F., M.Z.-C.; formal analysis: É.C., M.E., M.T.H., F.R., A.F., M.Z.-C.; writing—original draft preparation: É.C., M.E.; writing-review and editing: É.C., M.E., M.T.H., F.R., A.F., M.Z.-C.; visualization: É.C., M.E., M.T.H., F.R., A.F., M.Z.-C.; supervision: M.T.H., F.R.; project administration: M.T.H., F.R.; funding acquisition: M.T.H. and F.R. All authors have read and agreed to the published version of the manuscript.

Funding: This research was funded by the project, PTDC/SAU-SER/31678/2017, supported by the operational program on competitiveness and internationalization (POCI) in its FEDER/FNR component, POCI-01-0145-FEDER-031678, and by the Foundation for Science and Technology in its state budget component (OE).

Acknowledgments: We thank Tânia Magalhães Silva, (iBiMED/UA) for her collaboration in the revision of the manuscript.

Conflicts of Interest: The authors declare no conflict of interest, and the funders had no role in the design of the study; in the collection, analyses, or interpretation of data; in the writing of the manuscript, or in the decision to publish the results.

\section{References}

1. Mohr, K.I. History of Antibiotics Research. Curr. Top. Microbiol. Immunol. 2016, 398, 237-272. [CrossRef] [PubMed]

2. Goossens, H.; Ferech, M.; Vander Stichele, R.; Elseviers, M. Outpatient antibiotic use in Europe and association with resistance: A cross-national database study. Lancet 2005, 365, 579-587. [CrossRef]

3. Aslam, B.; Wang, W.; Arshad, M.I.; Khurshid, M.; Muzammil, S.; Rasool, M.H.; Nisar, M.A.; Alvi, R.F.; Aslam, M.A.; Qamar, M.U.; et al. Antibiotic resistance: A rundown of a global crisis. Infect. Drug Resist. 2018, 11, 1645-1658. [CrossRef]

4. Shively, N.R.; Buehrle, D.J.; Clancy, C.J.; Decker, B.K. Prevalence of Inappropriate Antibiotic Prescribing in Primary Care Clinics within a Veterans Affairs Health Care System. Antimicrob. Agents Chemother. 2018, 62, 1-7. [CrossRef] [PubMed]

5. Hulscher, M.E.; Grol, R.P.; van der Meer, J.W. Antibiotic prescribing in hospitals: A social and behavioural scientific approach. Lancet Infect. Dis. 2010, 10, 167-175. [CrossRef]

6. Forum of International Respiratory Societies. Respiratory Diseases in the World Realities of Today-Opportunities for Tomorrow, 1st ed.; European Respiratory Society: Sheffield, UK, 2013; ISBN 9781849840569. Available online: https://www.theunion.org/what-we-do/publications/technical/english/FIRS_report_for_web.pdf (accessed on 24 June 2020).

7. Pattemore, P.K.; Jennings, L.C. Epidemiology of Respiratory Infections. In Pediatric Respiratory Medicine; Elsevier: Amsterdam, The Netherlands, 2008; pp. 435-452; ISBN 9780323040488. Available online: https: //www.ncbi.nlm.nih.gov/pmc/articles/PMC7151775/ (accessed on 24 June 2020).

8. Brenda Tesini Overview of Viral Respiratory Infections-Infectious Diseases-MSD Manual Professional Edition. Available online: https://www.msdmanuals.com/professional/infectious-diseases/respiratoryviruses/overview-of-viral-respiratory-infections (accessed on 23 May 2020).

9. Sahadulla, M. Infections of the Respiratory System. In Concise Handbook of Infectious Diseases; Jaypee Brothers Medical Publishers (P) Ltd.: New Delhi, India, 2018; ISBN 0963117211. Available online: https: //www.jaypeedigital.com/book/9789386261939/chapter/ch11 (accessed on 23 May 2020).

10. World Health Organization. 2019 Antibacterial Agents in Clinical Development-an Analysis of the Antibacterial Clinical Development Pipeline; World Health Organization: Geneva, Switzerland, 2019; ISBN 9789240000193. Available online: https://apps.who.int/iris/bitstream/handle/10665/330420/9789240000193-eng.pdf (accessed on 27 May 2020). 
11. Kraus, E.M.; Pelzl, S.; Szecsenyi, J.; Laux, G. Antibiotic prescribing for acute lower respiratory tract infections (LRTI) - guideline adherence in the German primary care setting: An analysis of routine data. PLoS ONE 2017, 12, e0174584. [CrossRef]

12. Velickovski, F.; Ceccaroni, L.; Roca, J.; Burgos, F.; Galdiz, J.B.; Marina, N.; Lluch-Ariet, M. Clinical Decision Support Systems (CDSS) for preventive management of COPD patients. J. Transl. Med. 2014, 12, S9. [CrossRef]

13. dos Santos, M.A.F.R.N.; Tygesen, H.; Eriksson, H.; Herlitz, J. Clinical decision support system (CDSS)-effects on care quality. Int. J. Health Care Qual. Assur. 2014, 27, 707-718. [CrossRef]

14. Sutton, R.T.; Pincock, D.; Baumgart, D.C.; Sadowski, D.C.; Fedorak, R.N.; Kroeker, K.I. An overview of clinical decision support systems: Benefits, risks, and strategies for success. NPJ Digit. Med. 2020, 3, 17. [CrossRef]

15. Kawamoto, K.; Houlihan, C.A.; Balas, E.A.; Lobach, D.F. Improving clinical practice using clinical decision support systems: A systematic review of trials to identify features critical to success. BMJ 2005, 330, 765. [CrossRef]

16. Kannry, J.; McCullagh, L.; Kushniruk, A.; Mann, D.; Edonyabo, D.; McGinn, T. A Framework for Usable and Effective Clinical Decision Support: Experience from the iCPR Randomized Clinical Trial. eGEMs 2015, 3, 10. [CrossRef] [PubMed]

17. Sim, I.; Gorman, P.; Greenes, R.A.; Haynes, R.B.; Kaplan, B.; Lehmann, H.; Tang, P.C. Clinical Decision Support Systems for the Practice of Evidence-based Medicine. J. Am. Med. Informatics Assoc. 2001, 8, 527-534. [CrossRef] [PubMed]

18. Lehmann, C.U.; Séroussi, B.; Jaulent, M.-C. Troubled Waters: Navigating Unintended Consequences of Health Information Technology. Yearb. Med. Inform. 2016, 25, 5-6. [CrossRef] [PubMed]

19. SPMS-Serviços Partilhados do Ministério da Saúde. eHealth em Portugal-Visão 2020; SPMS: Lisboa, Portugal, 2015. Available online: http://spms.min-saude.pt/wp-content/uploads/2015/11/Relatório-ThinkTank-eHealth-2020-v05112015.pdf (accessed on 4 June 2020).

20. Bremmer, D.N.; Trienski, T.L.; Walsh, T.L.; Moffa, M.A. Role of Technology in Antimicrobial Stewardship. Med. Clin. North Am. 2018, 102, 955-963. [CrossRef]

21. PRISMA PRISMA Statement-Checklist. Available online: http://www.prisma-statement.org/ PRISMAStatement/Checklist (accessed on 27 May 2020).

22. Carvalho, É.F.S.; Estrela, M.; Figueiras, A.; Roque, F.; Herdeiro, M.T. eHealth Tools to Improve Antibiotic Use and Resistances: A Systematic Review. PROSPERO 2020 CRD42020167316. Available online: https: //www.crd.york.ac.uk/prospero/display_record.php?RecordID=167316 (accessed on 4 June 2020).

23. Carracedo-Martinez, E.; Gonzalez-Gonzalez, C.; Teixeira-Rodrigues, A.; Prego-Dominguez, J.; Takkouche, B.; Herdeiro, M.T.; Figueiras, A. Computerized Clinical Decision Support Systems and Antibiotic Prescribing: A Systematic Review and Meta-analysis. Clin. Ther. 2019, 41, 552-581. [CrossRef]

24. Garg, A.X.; Adhikari, N.K.J.; McDonald, H.; Rosas-Arellano, M.P.; Devereaux, P.J.; Beyene, J.; Sam, J.; Haynes, R.B. Effects of computerized clinical decision support systems on practitioner performance and patient outcomes: A systematic review. J. Am. Med. Assoc. 2005, 293, 1223-1238. [CrossRef]

25. Bourgeois, F.C.; Linder, J.; Johnson, S.A.; Co, J.P.T.; Fiskio, J.; Ferris, T.G. Impact of a computerized template on antibiotic prescribing for acute respiratory infections in children and adolescents. Clin. Pediatr. (Phila). 2010, 49, 976-983. [CrossRef]

26. Gifford, J.; Vaeth, E.; Richards, K.; Siddiqui, T.; Gill, C.; Wilson, L.; DeLisle, S. Decision support during electronic prescription to stem antibiotic overuse for acute respiratory infections: A long-term, quasi-experimental study. BMC Infect. Dis. 2017, 17, 528. [CrossRef]

27. Linder, J.A.; Schnipper, J.L.; Tsurikova, R.; Yu, T.; Volk, L.A.; Melnikas, A.J.; Palchuk, M.B.; Olsha-Yehiav, M.; Middleton, B. Documentation-based clinical decision support to improve antibiotic prescribing for acute respiratory infections in primary care: A cluster randomised controlled trial. Inform. Prim. Care 2009, 17, 231-240. [CrossRef]

28. Linder, J.A.; Bates, D.W.; Williams, D.H.; Connolly, M.A.; Middleton, B. Acute infections in primary care: Accuracy of electronic diagnoses and electronic antibiotic prescribing. J. Am. Med. Inform. Assoc. 2006, 13, 61-66. [CrossRef] 
29. Linder, J.; Schnipper, J.L.; Volk, L.A.; Tsurikova, R.; Palchuk, M.; Olsha-Yehiav, M.; Melnikas, A.J.; Middleton, B. Clinical decision support to improve antibiotic prescribing for acute respiratory infections: Results of a pilot study. AMIA Annu. Symp. Proc. 2007, 468-472.

30. Litvin, C.B.; Ornstein, S.M.; Wessell, A.M.; Nemeth, L.S.; Nietert, P.J. Use of an electronic health record clinical decision support tool to improve antibiotic prescribing for acute respiratory infections: The ABX-TRIP study. J. Gen. Intern. Med. 2013, 28, 810-816. [CrossRef] [PubMed]

31. Mainous, A.G., 3rd; Lambourne, C.A.; Nietert, P.J. Impact of a clinical decision support system on antibiotic prescribing for acute respiratory infections in primary care: Quasi-experimental trial. J. Am. Med. Inform. Assoc. 2013, 20, 317-324. [CrossRef] [PubMed]

32. Mann, D.; Knaus, M.; McCullagh, L.; Sofianou, A.; Rosen, L.; McGinn, T.; Kannry, J. Measures of user experience in a streptococcal pharyngitis and pneumonia clinical decision support tools. Appl. Clin. Inform. 2014, 5, 824-835. [CrossRef] [PubMed]

33. McCullagh, L.J.J.; Sofianou, A.; Kannry, J.; Mann, D.M.M.; McGinn, T.G.G. User centered clinical decision support tools: Adoption across clinician training level. Appl. Clin. Inform. 2014, 5, 1015-1025. [CrossRef]

34. McCullough, J.M.; Zimmerman, F.J.; Rodriguez, H.P. Impact of clinical decision support on receipt of antibiotic prescriptions for acute bronchitis and upper respiratory tract infection. J. Am. Med. Inform. Assoc. 2014, 21, 1091-1097. [CrossRef]

35. McDermott, L.; Yardley, L.; Little, P.; van Staa, T.; Dregan, A.; McCann, G.; Ashworth, M.; Gulliford, M. Process evaluation of a point-of-care cluster randomised trial using a computer-delivered intervention to reduce antibiotic prescribing in primary care. BMC Health Serv. Res. 2014, 14, 594. [CrossRef]

36. McGinn, T.G.; McCullagh, L.; Kannry, J.; Knaus, M.; Sofianou, A.; Wisnivesky, J.P.; Mann, D.M. Efficacy of an evidence-based clinical decision support in primary care practices: A randomized clinical trial. JAMA Intern. Med. 2013, 173, 1584-1591. [CrossRef]

37. Ginzburg, R.; Conway, J.J.; Waltermaurer, E.; Song, W.; Jellinek-Cohen, S.P. Using Clinical Decision Support Within the Electronic Health Record to Reduce Incorrect Prescribing for Acute Sinusitis. J. Patient-Cent. Res. Rev. 2018, 5, 196-203. [CrossRef]

38. Rattinger, G.B.; Mullins, C.D.; Zuckerman, I.H.; Onukwugha, E.; Walker, L.D.; Gundlapalli, A.; Samore, M.; Delisle, S. A sustainable strategy to prevent misuse of antibiotics for acute respiratory infections. PLoS ONE 2012, 7, e51147. [CrossRef]

39. Rubin, M.A.; Bateman, K.; Donnelly, S.; Stoddard, G.J.; Stevenson, K.; Gardner, R.M.; Samore, M.H. Use of a personal digital assistant for managing antibiotic prescribing for outpatient respiratory tract infections in rural communities. J. Am. Med. Inform. Assoc. 2006, 13, 627-634. [CrossRef] [PubMed]

40. Webb, B.J.; Sorensen, J.; Mecham, I.; Buckel, W.; Ooi, L.; Jephson, A.; Dean, N.C. Antibiotic Use and Outcomes after Implementation of the Drug Resistance in Pneumonia Score in ED Patients With Community-Onset Pneumonia. Chest 2019, 156, 843-851. [CrossRef] [PubMed]

41. Gonzales, R.; Anderer, T.; McCulloch, C.E.; Maselli, J.H.; Bloom, F.J.J.; Graf, T.R.; Stahl, M.; Yefko, M.; Molecavage, J.; Metlay, J.P. A cluster randomized trial of decision support strategies for reducing antibiotic use in acute bronchitis. JAMA Intern. Med. 2013, 173, 267-273. [CrossRef]

42. Graham, T.A.D.; Bullard, M.J.; Kushniruk, A.W.; Holroyd, B.R.; Rowe, B.H. Assessing the sensibility of two clinical decision support systems. J. Med. Syst. 2008, 32, 361-368. [CrossRef]

43. Grayson, M.L.; Melvani, S.; Kirsa, S.W.; Cheung, S.; Korman, A.M.; Garrett, M.K.; Thomson, W.A. Impact of an electronic antibiotic advice and approval system on antibiotic prescribing in an Australian teaching hospital. Med. J. Aust. 2004, 180, 455-458. [CrossRef]

44. Gulliford, M.C.; van Staa, T.; Dregan, A.; McDermott, L.; McCann, G.; Ashworth, M.; Charlton, J.; Little, P.; Moore, M.V.; Yardley, L. Electronic health records for intervention research: A cluster randomized trial to reduce anti biotic prescribing in primary care (eCRT study). Ann. Fam. Med. 2014, 12, 344-351. [CrossRef]

45. Gulliford, M.C.; Prevost, A.T.; Charlton, J.; Juszczyk, D.; Soames, J.; McDermott, L.; Sultana, K.; Wright, M.; Fox, R.; Hay, A.D.; et al. Effectiveness and safety of electronically delivered prescribing feedback and decision support on antibiotic use for respiratory illness in primary care: REDUCE cluster randomised trial. BMJ 2019, 364, 1236. [CrossRef]

46. Hingorani, R.; Mahmood, M.; Alweis, R. Improving antibiotic adherence in treatment of acute upper respiratory infections: A quality improvement process. J. Community Hosp. Intern. Med. Perspect. 2015, 5, 27472. [CrossRef] 
47. Jones, B.E.; Haroldsen, C.; Madaras-Kelly, K.; Goetz, M.B.; Ying, J.; Sauer, B.; Jones, M.M.; Leecaster, M.; Greene, T.; Fridkin, S.K.; et al. In Data We Trust? Comparison of Electronic Versus Manual Abstraction of Antimicrobial Prescribing Quality Metrics for Hospitalized Veterans with Pneumonia. Med. Care 2018, 56, 626-633. [CrossRef]

48. Holstiege, J.; Mathes, T.; Pieper, D. Effects of computer-aided clinical decision support systems in improving antibiotic prescribing by primary care providers: A systematic review. J. Am. Med. Informatics Assoc. 2015, 22, 236-242. [CrossRef]

49. Baysari, M.T.; Lehnbom, E.C.; Li, L.; Hargreaves, A.; Day, R.O.; Westbrook, J.I. The effectiveness of information technology to improve antimicrobial prescribing in hospitals: A systematic review and meta-analysis. Int. J. Med. Inform. 2016, 92, 15-34. [CrossRef] [PubMed]

50. Shebl, N.A.; Franklin, B.D.; Barber, N. Clinical decision support systems and antibiotic use. Pharm. World Sci. 2007, 29, 342-349. [CrossRef] [PubMed]

51. Rawson, T.M.; Moore, L.S.P.; Hernandez, B.; Charani, E.; Castro-Sanchez, E.; Herrero, P.; Hayhoe, B.; Hope, W.; Georgiou, P.; Holmes, A.H. A systematic review of clinical decision support systems for antimicrobial management: Are we failing to investigate these interventions appropriately? Clin. Microbiol. Infect. 2017, 23, 524-532. [CrossRef] [PubMed]

52. Granja, C.; Janssen, W.; Johansen, M.A. Factors Determining the Success and Failure of eHealth Interventions: Systematic Review of the Literature. J. Med. Internet Res. 2018, 20, e10235. [CrossRef] [PubMed]

53. Silva, A.G.; Simões, P.; Santos, R.; Queirós, A.; Rocha, N.P.; Rodrigues, M. A scale to assess the methodological quality of studies assessing usability of electronic health products and services: Delphi study followed by validity and reliability testing. J. Med. Internet Res. 2019, 21, e14829. [CrossRef] [PubMed]

54. Horsky, J.; Schiff, G.D.; Johnston, D.; Mercincavage, L.; Bell, D.; Middleton, B. Interface design principles for usable decision support: A targeted review of best practices for clinical prescribing interventions. J. Biomed. Inform. 2012, 45, 1202-1216. [CrossRef]

55. Sirajuddin, A.M.; Osheroff, J.A.; Sittig, D.F.; Chuo, J.; Velasco, F.; Collins, D.A. Implementation pearls from a new guidebook on improving medication use and outcomes with clinical decision support. Effective CDS is essential for addressing healthcare performance improvement imperatives. J. Healthc. Inf. Manag. 2009, 23, 38-45.

56. Peters, D.H.; Tran, N.T.; Adam, T. Implementation Research in Health-A Practical Guide; World Health Organization: Geneva, Switzerland, 2013; ISBN 9789241506212. Available online: https://apps.who.int/iris/bitstream/handle/10665/91758/9789241506212_eng.pdf;jsessionid= 8EA86C567F2C099387E235828FC55D35? sequence=1 (accessed on 3 July 2020).

57. Ferri, M.; Ranucci, E.; Romagnoli, P.; Giaccone, V. Antimicrobial resistance: A global emerging threat to public health systems. Crit. Rev. Food Sci. Nutr. 2017, 57, 2857-2876. [CrossRef] 